\title{
New Insights into Molecular Diagnostics for Common Gastrointestinal Infections
}

\author{
Varsha Gupta ${ }^{1}$, Meenakshi Singh ${ }^{2}$, Aditi $^{3}$, Ritu Garg $^{4}$
}

\begin{abstract}
Gastrointestinal (GI) infections are a major health problem all over the world, causing an increase in hospitalization, morbidity, and mortality. The etiological agents of infectious gastroenteritis are viruses, bacteria, and parasites. A precise identification of Gl pathogens is crucial for proper treatment and/or isolation, management, and further investigations like designing specific prevention modalities, vaccination strategies, and empiric treatment regimens to prevent the spread of the infectious agents. Routinely, the laboratory diagnosis of Gl infections depends on microscopy, culture, and antigen detection. The drawbacks of conventional method are its low sensitivity and 3-5 days of turnaround time in the finalization of report. Quick turnaround time is of paramount value in diagnosis, clinical management, and infection control. From the last decade, molecular-based diagnostic tools have emerged for Gl infections in the microbiological laboratory analyses. Culture-independent diagnostic tests typically involve nucleic acid amplification of the genetic material of several bacteria, viruses, and parasites simultaneously. Even whole-genome next-generation sequencing is important for symptomatic patients that remain negative by both routine and multiplex PCR-based diagnostic methods. Therefore, the use of proficient methods for pathogen detection is necessary to ensure prompt turnaround time. This review includes various conventional and molecular tools in identifying various enteropathogens and also analyzes the advantages and drawbacks of all methods.
\end{abstract}

Keywords: Culture, Diarrhea, Enteropathogens, Gastrointestinal infections, Real-time PCR.

Journal of Gastrointestinal Infections (2021): 10.5005/jp-journals-10068-3044

\section{INTRODUCTION}

Gastrointestinal (GI) infections remain an unmet challenge in global health. Gl infections can be categorized as gastritis, enteritis, and gastroenteritis. An inflammation of the protective lining of the stomach is known as gastritis, and it is further classified into acute and chronic gastritis. ${ }^{1-3}$ Enteritis is the inflammation of the small intestine part only. Gastroenteritis involves both inflammation of the stomach and the intestine and is also known as infectious diarrhea, which is the major illness related to GI infections. Diarrhea is defined as passage of three or more loose stools in a day. ${ }^{4}$ Worldwide, diarrhea leads to a number of outpatient visits, inpatient load, and loss of quality of life, in both domestic settings and among people traveling abroad. It has been estimated that 4-6 million children die each year of diarrheal diseases, mainly in developing countries of Asia and Africa. ${ }^{5}$ Developed countries like United States have reported infectious enteritis and foodborne illness in around 1.3 million patients diagnosed with enteritis or Gl symptoms. ${ }^{6}$

Accurate detection of GI pathogens is crucial for appropriate management, treatment and/or isolation, as well as further investigations like designing specific prevention modalities, vaccination strategies, and empiric treatment regimens to prevent the spread of the infectious agents. Rapid turnaround time is also imperative for clinical management, diagnosis, and infection control, and consequently, the use of effective methods for pathogen detection is necessary, which decreases the turnaround time. The challenge in evaluating a patient is to decide what measures to follow that will lead to a most direct and efficient diagnosis. There are different diagnostic modalities required for evaluating patients with Gl illness, which are laboratory studies, endoscopy, and diagnostic imaging. Advancement in these three areas has provided clinicians with a medley of testing modalities
${ }^{1-3}$ Department of Microbiology, Government Medical College and Hospital, Chandigarh, India

${ }^{4}$ Department of Microbiology, Maharishi Markandeshwar Institute of Medical Sciences and Research (MMIMSR), Mullana, Haryana, India

Corresponding Author: Ritu Garg, Department of Microbiology, Maharishi Markandeshwar Institute of Medical Sciences and Research (MMIMSR), Mullana, Haryana, India, Phone: +91 9872885319, e-mail: dr_rittu07@yahoo.co.in

How to cite this article: Gupta V, Singh M, Aditi, et al. New Insights into Molecular Diagnostics for Common Gastrointestinal Infections. J Gastrointest Infect 2021;11:15-23.

Source of support: Nil

Conflict of interest: None

at their fingertips. This article reviews and compares both conventional and molecular-based laboratory tests, and advantages and limitations of diagnostics for common Gl infections.

\section{Etiological Agents of Infectious Gastroenteritis}

Diarrhea due to viral and bacterial infections is a crucial public health problem especially in developing countries. A wide range of enteric pathogens can cause gastroenteritis. The causative agents of infectious diarrhea may vary according to geographical locales, urban to rural areas, and depend on a few factors such as comorbidities and host immune status. However, the most common among these agents are viral pathogens, especially in children up to 9 years. Parasitic infections are also an important cause, particularly in a tropical country like India. Bacterial causes are more responsible for severe cases of infectious diarrhea compared 
to other infections. Ironically, the routine processing tests available for bacterial pathogens are more than those for viral pathogens.

- Viral gastroenteritis: It has been estimated that $4-38 \%$ of deaths among children $<5$ years of age are caused by viral infections. Viruses, including Adenovirus (enteric types 40 and 41), Astrovirus, Coxsackie virus, Norovirus, Rotavirus, and Sapovirus, are substantiated to be the most common causative agents. In current years, numerous novel enteric viruses such as Aichivirus, Kobuvirus, Enteroviruses, Parechoviruses, Salivirus, Parechoviruses (family Picornaviridae), and human bocaviruses (family Parvoviridae) have also been found to be associated with acute gastroenteritis. ${ }^{7}$

- Bacterial gastroenteritis: Bacterial pathogens like nontyphoidal Salmonellae and Campylobacter spp. are the most common cause of severe bacterial infections in the United States. Other bacterial causes include Clostridium perfringens and enterotoxigenic Escherichia coli (ETEC), which cause watery diarrhea. The list of diarrheagenic pathogens is extensive and includes Bacillus cereus, Campylobacter spp., Clostridium difficile (toxigenic), C. perfringens, Shiga toxin-producing E. coli (STEC), E. coli O157:H7, ETEC, diarrheagenic E. coli other than STEC and ETEC, Helicobacter pylori, Listeria monocytogenes, Plesiomonas shigelloides, Shigella spp., Salmonella enterica nontyphi, S. enterica serotype Typhi, Vibrio and Vibrio-like spp., Staphylococcus aureus, Yersinia enterocolitica, and other species. ${ }^{8}$

- Parasitic infections: The infections caused by parasites can present with different clinical manifestations, depending on the agent and various host factors. They result in more severe infections in immunocompromised individuals. These infections can cause enteritis, diarrhea, dysentery (Giardia lamblia, Cryptosporidium parvum or C. hominis, and Entamoeba histolytica, etc.), invasive disease (E. histolytica and Balantidium coli), nutritional depletion (Cryptosporidium, Cystoisospora, G. lamblia, Ancylostoma, Necator americanus, etc.), and mechanical obstruction (Ascaris). ${ }^{9}$

\section{Conventional Approaches for GI Pathogens}

By tradition, the laboratory diagnosis of $\mathrm{Gl}$ infections relies on microscopy, culture, and antigen detection. The stool culture still remains the gold standard for identifying bacterial enteropathogens, even though it has a relatively low sensitivity and is laborious. ${ }^{10}$ Staining techniques also help in the detection of bacterial pathogens as well. For bacterial culture, a delay in transport or processing can lead to a decreased viability of certain pathogens, including Campylobacter and Vibrio spp., unless transported in a transport medium like Cary-Blair. The advantages of culture method include its specificity for the pathogenic organism isolated in patient and the availability of the isolate for antibiotic sensitivity testing. ${ }^{11}$ Additionally, the strains isolated can be referred to state public health laboratories for further identification, epidemiological studies, or outbreak investigations. The drawbacks of this conventional approach are its low sensitivity and 3-5 days of turnaround time in the finalization of report. For virus cultures, the specimen should be refrigerated if not inoculated into cell cultures within 2 hours. Apart from microscopy and culture, antigen and antibody detection can be done by tests like ELISA. Enzyme immunoassays can be used to detect numerous microorganisms, which cause GI infections like E. coli O157:H7 and
Campylobacter spp., the presence of the Shiga toxins produced by STEC, or the presence of $C$. difficile toxins $A$ or $A$ and $B .{ }^{12}$ Furthermore, antigen tests are also available to detect some viruses causing gastroenteritis, such as rotavirus and adenovirus; limitations of the same are that these tests show a variable sensitivity and are not available for all enteropathogens.

For parasitic infections, concentration techniques, indirect wet mount, and permanently stained smear can be employed for the detection of ova and parasites. Additionally, antigen tests are also available to detect certain specific parasites such as G. lamblia, Cryptosporidium spp., or E. histolytica. Infectious inflammatory and secretory diarrhea can also be differentiated on the basis of the presence of leukocytes in case of inflammatory diarrhea. ${ }^{13}$

\section{Emerging Molecular Approaches}

From the last decade, molecular-based diagnostic tools have emerged for $\mathrm{Gl}$ infections in the microbiological laboratory. Culture-independent diagnostic tests typically involve nucleic acid amplification of the genetic material of several bacteria, viruses, and parasites simultaneously. This not only allows for a rapid diagnosis of previously difficult-to-detect and culture pathogens, including several bacteria, viruses, and parasites, and is not limited to $E$. coli 0157, Salmonella spp., ETEC, Norovirus, and Giardia.

Culture-independent diagnostic tests include:

- Singleplex/monoplex techniques and multiplex molecular assays

- Microfluidics and array technologies

- Fully automatic platforms in which a single-step nucleic acid extraction, amplification, and analysis are done.

Of late, isothermal amplification has also gained popularity in which no expensive thermal cycling equipment pieces are required and isothermal helicase-dependent amplification can detect a single pathogen at a time. ${ }^{14}$

\section{Real-time Polymerase Chain Reaction}

Real-time PCR can detect various organisms such as Shigella spp., Salmonella spp., Campylobacter spp., and various diarrheagenic E. coli strains often with better sensitivity compared to bacterial culture. ${ }^{15}$ The selection of detection method is the basis for efficient diagnosis since each method has different sensitivity and specificity. Real-time PCR assays have good performance, but are labor-intensive and are time-consuming. Molecular tests for various GI pathogens are commercially available in the form of real-time $P C R$, which can be singleplex or multiplex. These days, multiplex molecular assays based on PCR methodology are applied for the detection and identification of pathogens responsible for causing diarrhea and other infectious diseases. ${ }^{16}$ These syndromic panels permit healthcare providers to achieve a timely diagnosis, which is crucial in certain patient populations, like immunocompromised hosts and the critically ill patients by allowing the diagnosis of a wide range of pathogens. ${ }^{17}$

Singleplex assays are used for a single pathogen like in the case of $C$. difficile. Being one of the most important pathogens causing antibiotic-associated diarrhea particularly in hospitalized patients, several molecular platforms are available for it. ${ }^{18}$ The virulent genes of this pathogen are present on pathogenicity island covering toxigenic genes: toxin A, toxin B, and binary toxins. ${ }^{19}$ In the last decade, several outbreaks have occurred due to the hypervirulent strain of C. difficile. ${ }^{20}$ According to Infectious Diseases Society of 
America/Society for Healthcare Epidemiology of America (IDSA/ SHEA), C. difficile testing is suggested with onset of $\geq 3$ unformed stools in 24 hours in high-risk adults and children of $\geq 2$ years of age following antimicrobial treatment, in healthcare-associated diarrhea, and in patients with persistent chronic diarrhea without any etiology. ${ }^{21}$ Several FDA-approved platforms are available for C. difficile toxin identification: Illumigene ${ }^{\circledast} \mathrm{C}$. difficile targeting $t c d A$ and $t c d B$ (Meridian Bioscience, Milan, Italy), PCRFast ${ }^{\oplus}$ C. difficile $A / B$ targeting $t c d A$ and $t c d B$ (GmbH, Berlin, Germany), GeneXpert ${ }^{\circledR}$ C. difficile/Epi targeting $t c d A, t c d B, \Delta 117 t c d C$ and $c d t$ (Cepheid, Sunnyvale, California, USA) etc are available. This not only helps in specific detection but also detects 027 and 066/078 C. difficile, which are hypervirulent ribotypes (GeneXpert ${ }^{\circledR}$ C. difficile/Epi and in-house $\mathrm{PCR}$ ) leading to better patient care as well as timely epidemiological control measure application. ${ }^{22}$

Another important organism causing Gl illness is norovirus. The most frequently used modality for its diagnosis is RT-PCR, like RIDAGENE Norovirus (R-Biopharm, Darmstadt, Germany) and AccuPower Norovirus Real-time RT-P CR Kit (Bioneer Co., Daejeon, South Korea). These PCR assays detect Noroviruses GI/GII. ${ }^{23}$ Genogroup IV has been recently included in some PCR assays as it also causes acute gastroenteritis. ${ }^{24}$

However, there are a wide range of pathogens that have nondistinguishable clinical presentations caused by GI infections; therefore, the identification of multiple organisms is a more efficient approach for appropriate management. There are several FDA- or CE-IVD- approved open and closed multiplex commercial systems that identify the most common pathogens. ${ }^{25}$ These assays are helpful in the detection of multiple enteropathogens especially in relation to local epidemiology and prevalence. Thus, before acquisition of these investigations, institutional need should be considered. Presently, 11 FDA-approved multiplex assays are available for enteric pathogens. These assays not only detect multiple microorganisms at one fell swoop but also select resistance genes that are important for therapy and management.

- Verigene Enteric Pathogens Test: It is manufactured by Luminex Corporation (Luminex, Austin, USA). This system received FDA approval/clearance in 2012. It is an integrated system that can detect five bacterial (Campylobacter spp., Salmonella spp., Shigella spp., Vibrio spp., Y. enterocolitica, and Shiga toxins 1 and 2) and two viral (Norovirus and Rotavirus) pathogens. This panel cannot detect parasites. This platform uses a processor and a reader that can simultaneously perform nucleic acid extraction, amplification, and hybridization to probes on a glass slide in a microarray format. However, a comparative study done on three different platforms, viz., Verigene EP test, BioFire FilmArray GI panel, and Luminex xTAG Gl panel, showed this technique to be less sensitive and specific as compared to BioFire Array GI panel. ${ }^{26}$

- BioFire FilmArray: It is manufactured by BioFire Diagnostics, LLC (BioFire, USA), which got FDA approval in 2013. Film array is a fully automatic multiplex PCR system, which simultaneously performs nucleic acid extraction, reverse transcription, amplification, and analysis within 1 hour. It has a long list of bacterial, viral as well as parasitic pathogens that can be identified by it, including E. histolytica, Cyclospora cayetanensis, Y. enterocolitica, etc. The advantages of this system are a comprehensive coverage of many of the major enteropathogens and rapid turnaround time. ${ }^{17}$ In a multicenter study, a comparison of BioFire GI Panel, conventional stool culture, and molecular methods was done, which showed the FilmArray GI Panel sensitivity as $100 \%$ for 12 of the 22 and $>94.5 \%$ for an additional 7 of the 22 target pathogens tested. For the rest of the three targets due to the low prevalence of the pathogens in the study, sensitivity could not be calculated. ${ }^{27}$ In another retrospective cohort study done in immunocompromised patients, 124 patient samples were tested positive by BioFire GI Panel, compared with 45 patient specimens by conventional testing. ${ }^{17}$ The automatic panel group as compared to conventional method was found to be highly advantageous as it demonstrated a higher co-infection rate (48.4 vs $13.3 \%$ ) and quicker turnaround time (23.4 vs 71.4 hours). Moreover, this panel was also able to identify 29 potential viral infections that were undetectable by conventional stool tests, which warrants against unnecessary prescription of antibiotics.

- xTAG GPP Panel: This syndromic panel manufactured by Luminex Corporation (Luminex, Austin, USA) got FDA approval in 2014. This multiplex RT-PCR assay detects 15 enteropathogens, including 5 viruses (Adenovirus 40/41, Norovirus GI/II, Rotavirus A), 9 bacteria [Campylobacter, C. difficile, E. coli O157, ETEC (LT/ ST), STEC (stx1/stx2), Salmonella, Shigella, Vibrio cholera and $Y$. enterocolitica, and three parasites (Cryptosporidium spp., Giardia spp., and E. histolytica)]. This assay can detect enteropathogens with sensitivity ranging from 90 to $100 \%$, depending on pathogen present, and specificity between 91 and $99 \% .{ }^{28-30}$ This assay is helpful in identifying mixed infections requiring 5 hours for analysis and can process 96 samples at once. However, the disadvantages of this system is the requirement of separate nucleic acid extraction, and a high level of technical skill required to prevent cross-contamination as the operator must handle the PCR product before the hybridization step. In this system, the mean fluorescence intensity (MFI) is generated for each bead population and analyzed automatically by the xTAG Data Analysis Software GPP. Although Luminex is not designated as a quantitative assay, MFI values between consensus-positive and false-positive cases can be compared. ${ }^{31}$

- BD Max ${ }^{\mathrm{TM}}$ Enteric and Extended Bacterial Panel (BD Max EBP): As the name suggests, BD Max Enteric panel can detect bacterial pathogens only, which include Salmonella, Shigella, and Campylobacter, STEC (stx1/stx2). However, the other system of BD, i.e., BD Max EBP, can detect Y. enterocolitica, ETEC, Vibrio, and $P$. shigelloides. The system is a walkaway microfluidic RT-PCR instrument manufactured by Dickinson (Becton Dickinson, USA). It can process 24 samples at once in 3 hours. The advantage is that it requires 2 minutes of hands-on time per sample and thus has less chances of contamination. Stool specimen is placed into the BD MAX sample buffer tubes and vortexed. The tubes along with the BD MAX enteric bacterial panel reagent strip are then loaded into the instrument. This is then processed using the multiplex PCR assay after preparing the sample followed by lysis and extraction of the nucleic acid through an automated process. In various studies comparing conventional methods and the BD Max EBP assay for the detection of enteric bacterial pathogens in stool specimens, the BD Max EBP assay demonstrated a higher sensitivity and excellent specificity. ${ }^{32,33}$

Apart from bacterial identification, this company also offers separate systems for parasites and viruses detection known as the BD Max enteric parasite panel (Max EPP), which detects G. lamblia, E. histolytica, and Cryptosporidium spp. (C. parvum and C. hominis). It has shown good specificity and sensitivity for all targets, whereas sensitivity for $G$. intestinalis was equivalent to microscopic detection 
with the BD Max enteric parasite panel. ${ }^{34}$ The BD Max enteric viral panel (Max EVP), which detects rotavirus type $A$, norovirus genogroup I (GI) and GIl, adenovirus type $F$ 40/41, human astrovirus, and sapovirus (genogroups I, II, IV, and V), has also come up in the market and has shown to be valuable for the differential diagnosis of enteric disease caused by these viruses. ${ }^{35}$

- Seegene Allplex Gastrointestinal Full Panel Assay (AGPA): This is a one-step reverse transcription real-time multiplex PCR assay manufactured by Seegene (Seegene, Seoul, South Korea), which is CE-IVD-approved. This system recognizes 13 bacteria, 6 parasites, and 5 viruses in four multiplex PCR panels (bacteria I, bacteria II, virus, and parasite). The nucleic acid is extracted separately after which the Microlab Nimbus IVD or CFX96 ${ }^{\mathrm{TM}}$ realtime PCR system (Bio-Rad Laboratories, Richmond, California, USA) automatically performs the nucleic acid processing and PCR setup. The principle used in this assay involves using novel analytical multiple detection temperature (MuDT) technique, thus perceiving multiple targets using a single fluorescence channel without the use of melting curve analysis. After the setup, fluorescence is sensed at two temperatures $\left(60^{\circ} \mathrm{C}\right.$ and $72^{\circ} \mathrm{C}$ ). A distinct exponential fluorescence curve is observed beyond the crossing threshold at a value of less than 42 for the individual targets, if the result is positive. The four aforementioned panels can be selected according to the patient condition: virus and bacteria I panels could be used for hospitalized patients with a suspected nosocomial infection and bacteria I and II panels for patients having occult blood in stool.

Overall, this system had a $>2$-fold higher detection rate compared to conventional methods (44.4 vs $17.8 \%$ ) in a study done at Children's Hospital of Eastern Ontario, Canada. The study showed that norovirus genogroup II detection by the AGPA was higher in number, as it also detected the same in specimens which were negative by electron microscopy. Similarly, the bacterial pathogens, i.e., non-O157 STEC, enteropathogenic E. coli (EPEC), and enteroaggregative E. coli (EAEC), which were overlooked by conventional culture methods, were also evident by AGPA. ${ }^{36}$

In one study comparing Seegene, Luminex, and BD MAX for detecting GI pathogens, Seegene Allplex GI had the highest overall positive percent agreement (94\%; 258 of 275) and negative percent agreement (98\%; 571 of 583), respectively, while the BD MAX Enteric assay occupied second place with overall percentage agreement of $96 \%$ (362 of 379) in bacterial pathogen detection except $C$. difficile. Additionally, Seegene or Luminex was more sensitive for the detection of Campylobacter spp. as compared to BD MAX Enteric assay.

The company also offers an alternative multiplex PCR-based CE-IVD-approved kit named as Seeplex Diarrhea ACE Detection kits (Seegene, Seoul, Korea) with three assays (bacteria 1, bacterial 2, and virus) with the ability to simultaneously detect common bacterial and viral multipathogens including nine bacteria, four viruses, and a $C$. difficile toxin-producing gene. In this assay, independent nucleic acid extraction is needed, and then reverse transcription PCR is done and products formed are separated by capillary electrophoresis. The disadvantages include separate nucleic acid extraction and no detection of parasitic pathogens. There is a variation in sensitivity (40-100\%) and specificity (96-100\%) of these assays according to the pathogen in the sample. ${ }^{37-39}$

- ProGastro SSCS Assay: This is another commercially available FDA-approved kit (Hologic, San Diego, California, USA) used for the simultaneous qualitative detection of four bacterial pathogens [Campylobacter, Salmonella, Shigella, and STEC (stx1 and stx2 genes)]. As it is not an integrated system, it requires a separate nucleic acid extraction step, followed by PCR amplification in SmartCycler (Cepheid, Sunnyvale, California, USA) and data analysis. The overall sensitivity of this assay is $98.5 \%$ and specificity is in the range of $98.9-99.4 \%$, depending on the target pathogen. ${ }^{40,15}$

- RIDA Gene Real-time PCR Kits: These RT-PCR kits are CE-IVDapproved and manufactured by R-BioPharma (R-Biopharm AG, Darmstadt, Germany) that can detect various bacteria, viruses, and parasites simultaneously. A comparative study of seven different RT-PCR kits showed a less diagnostic capacity (PPA$81.7 \%$ ) of this kit, which could be due to failure in the detection of Campylobacter species other than C. jejuni and C. coli (C. upsaliensis, C. hyointestinalis, $C$. helveticus, or $C$. rectus) rather than in the sensitivity of the test, since most of the samples were in high concentration. ${ }^{41}$ However, in a different study comparing this kit with traditional methods for the detection of Campylobacter and Shigella species, the results of the kit were found to be more sensitive. ${ }^{42}$

- FTD Bacterial Gastrointestinal Panel: This FTD panel (Fast Track Diagnostics, Junglinster, Luxembourg) is a CE-IVD-cleared two-step multiplex RT-PCR test for the detection of pathogen genes by TaqMan technology. Being not integrated system, it requires separate nucleic acid extraction step. In this technique, the first tube performs multiplex detection of three species of C. coli/C. jejuni/C. lari and enterohemorrhagic E. coli (EHEC), while the second tube is used for detecting Salmonella spp., Shigella/ enteroinvasive E. coli, Y. enterocolitica, and C. difficile. A study by Biswas et al., ${ }^{42}$ evaluated and compared the diagnostic accuracy and the turnaround time of three multiplex molecular panels: the RIDA ${ }^{\oplus}$ GENE Bacterial Stool and EHEC/EPEC Panels, the FTD ${ }^{\circledR}$ Bacterial Gastroenteritis, and the BD MAX ${ }^{\mathrm{TM}}$ Enteric Bacterial Panel, suggesting all of the three as more sensitive as compared to conventional culture method by detecting additional 13 targets that were negative by culture. On comparing the turnaround time, all the three multiplex panels were much faster as compared to conventional technique ( $<3$ vs 66.5 hours).

- CLART EnteroBac Panel: There is one another two-tube PCR array-based molecular technique manufactured by Genomica (Genomica, Madrid, Spain) that simultaneously allows the detection of eight bacterial pathogens. The assay follows the steps for nucleic acid extraction and amplification, and detection is carried out on a low-density microarray analyzed by the company's CAR reader. Apart from the company itself, no validation has been done outside.

- QIAstat-Dx Gastrointestinal Panel: The QIAstat GIP (Qiagen, Hilden, Germany), an integrated multiplex PCR system, having a closed system for nucleic acid extraction, real-time PCR amplification, and fluorescent amplicon detection, uses cartridge and QIAstat-Dxanalyzer. It can detect and identify 24 gastroenteritis pathogens directly from stool samples in CaryBlair transport medium concurrently. A multicenter comparative study of QIAstat GIP with BioFire FilmArray GIP and Seegene Allplex GIP shows positive percent agreement of $98.2 \%$ and good correlation of QIAstat GIP with other assays. ${ }^{43}$

- GastroFinder 2SMART: The GastroFinder 2SMART assay (PathoFinder, the Netherlands), a CE-IVD-approved multiplex real-time PCR-based assay, can detect nine bacterial, five viral, and four parasitic enteropathogens but needs a separate nucleic acid extraction process, which is then followed by real-time 
PCR amplification and melting curve analysis based on the identification of organisms. Validation of this kit's performance is assessed by the manufacturer itself.

- EasyScreen Enteric Assay: This is non-FDA and non-CE-IVD kit (Genetic Signature's, Sydney, Australia), based on company's 3base technology that converts all cytosine bases $(C)$ in the starting nucleic acid samples to thymidine $(\mathrm{T})$ and results in a reduction in sequence variation. This allows for a greater number of multiplex targets to be run under similar conditions. There are separate panels for the detection of common bacterial, parasitic, viral pathogens, as well as $C$. difficile including hypervirulent 027 and 078 strains. In one of the studies, done by Stark et al., sensitivity was shown to be $92-100 \%$, specificity was $100 \%$, and the assay detected all commonly found subtypes and genotypes of clinically important human parasites. ${ }^{44}$

- Fecal Pathogens M Detection Assay: Aus Diagnostics Fecal Pathogens M detection assay (AusDiagnostics, Mascot, Australia) (non-FDA and non-CE-IVD) can detect 14 common bacterial, viral, and parasitic enteropathogens. This assay uses multiplexed tandem PCR technique comprising two amplification steps. In the first step, extracted nucleic acid is pre-amplified as a single-well multiplex reaction. The amplified product in the first step is then diluted, followed by the second step multiplex real-time PCR using SYBR green dye, and finally a melting curve analysis-based identification of organisms is done. Table 1 shows multiplex commercial tools with the list of pathogens, reported sensitivities, amount of automation, and pros and cons.

\section{Benefits and Drawbacks of Multiplex Gi Panels}

The overall advantages of multiplex panels include increased diagnostic yield, improved workflow, and reduced hands-on time as well as the important impact on infection control. One important benefit it provides is shorter hospital stay and reduction of antibiotic therapy. Also, these tests are more user-friendly, not needing highly trained personnel. Besides, several enteropathogens that have the capability to cause rapidly spreading outbreaks and epidemics can also be readily identified by these panels.

However, some microorganisms may not be clinically relevant such as those that can be shed in feces for several weeks. Similarly, some people may be asymptomatic carriers for certain organisms. Also, these methods cannot distinguish between viable and nonviable microorganisms. One major disadvantage of the molecular diagnostics is the unavailability of the isolates for further prospective studies. Another limitation of molecular-based testing is that antimicrobial testing cannot be performed by this method. Sometimes, the specimen collected for culture-independent testing might be incompatible with culture-based testing due to the use of inappropriate collection media used or inadequate method of collection.

The main current concern is cost as these tests cost much more than the conventional methods and at the same time the pathogens detected might not be clinically significant. Thus, the judicious use of multiplex panel is of paramount importance to diagnose the causative organism taking care not to overuse the drugs meant to treat these organisms, which create the problem of drug resistance in them.

\section{Next-generation Sequencing}

Next-generation sequencing (NGS) helps to sequence mixed populations of DNA or RNA genomes rapidly. This technique has found its application predominantly in areas where conventional diagnostic approaches present limitations, such as in understanding the epidemiology of many diarrhea-associated bacterial pathogens, identifying novel pathogens, and also identifying acknowledged pathogens. ${ }^{45}$ The etiology of suspected GI infections in acutely ill hospitalized or immunosuppressed patients usually remains undiagnosed, resulting in increased mortality and morbidity due to delayed or inadequate treatment, prolonged stays, or readmissions. In these types of patients, the identification of known or unknown pathogen is of utmost importance so as to start the correct therapy. NGS is also an effective approach for the detection of novel pathogens as well as to identify several putative diarrheal pathogens. In time, it is likely that probe-based detection will be taken over by sequencing, for the detection of unknown pathogens too. $^{46}$

Whole-genome next-generation sequencing (WG-NGS) is important for samples that are negative by both routine and multiplex PCR-based diagnostic methods while the patient remains symptomatic. It helps us to allow the identification of non-predefined targeted microorganisms, and it also allows enteric disease surveillance, thus helping to detect and investigate outbreaks and to monitor disease trends. The implementation depends on the ratio between costs and clinical benefits. Recently, origins of the Haitian cholera outbreak were analyzed using WG-NGS and phylogenetic analysis. ${ }^{47}$ However, there are few limitations with this technology: the cost is high, it takes time for diagnosis, and more importantly, it is unable to identify the causative agent in a large part of the samples. ${ }^{48}$

\section{CRISPR-Cas9 System}

C. difficile infection has become a grave health problem, which results in thousands of deaths all over the world annually. The dearth of genome engineering tools for $C$. difficile has delayed the machine-like understanding of the interaction between this pathogen and its hosts, as well as its pathology. Plasmids that carry the CRISPR-Cas9 system were created and conjugated into C. difficile. Colony PCR having primers that anneal to regions flanking the target gene deletion/integration locus was then used to identify the mutants, while heat-survival assay was done and comparison of the sporulation frequency between the mutant with spo0A deletion and the wild-type strain was observed. The resulting fluorescence in the mutant which has insertion of the green fluorescent protein (GFP) gene was then seen under a fluorescent microscope. This tool enabled the mutation efficiency of $100 \%$ for spo0A deletion. Conversely, required genes can be inserted into the $C$. difficile chromosome: an anaerobic GFP gene was inserted with a mutation efficiency of $80 \%$. This tool has the potential for the advancement of novel strategies for CDI diagnostics as well as therapies. ${ }^{49}$

After rotavirus, the second most important diarrheal pathogen is Cryptosporidium. It is also an important opportunistic pathogen in AIDS and organ transplant patients. Drug and vaccine development for cryptosporidiosis is restricted because of the poor traceability of this pathogen as there is deficiency of culture tools, inappropriate animal models, and molecular genetic tools. ${ }^{50}$ In such a scenario, CRISPR/Cas9 technology provides us with a valiant new era to help study this pathogen. The application of Cryptosporidium genetic modification will not only help us to increase our understanding of the basic biology of the parasite and its virulence, but will also help us in the development of upgraded vaccines and therapeutics. ${ }^{51}$ 


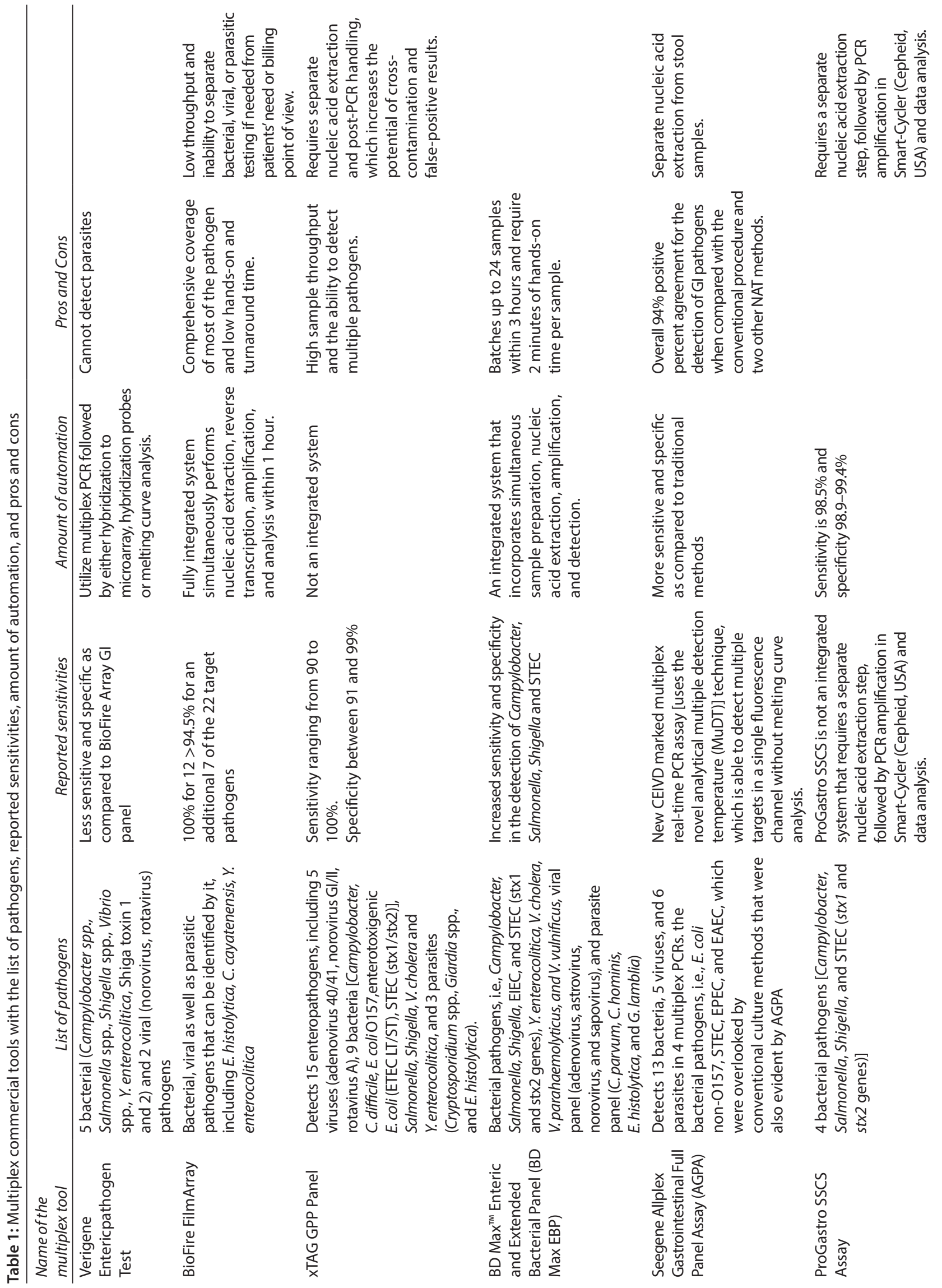



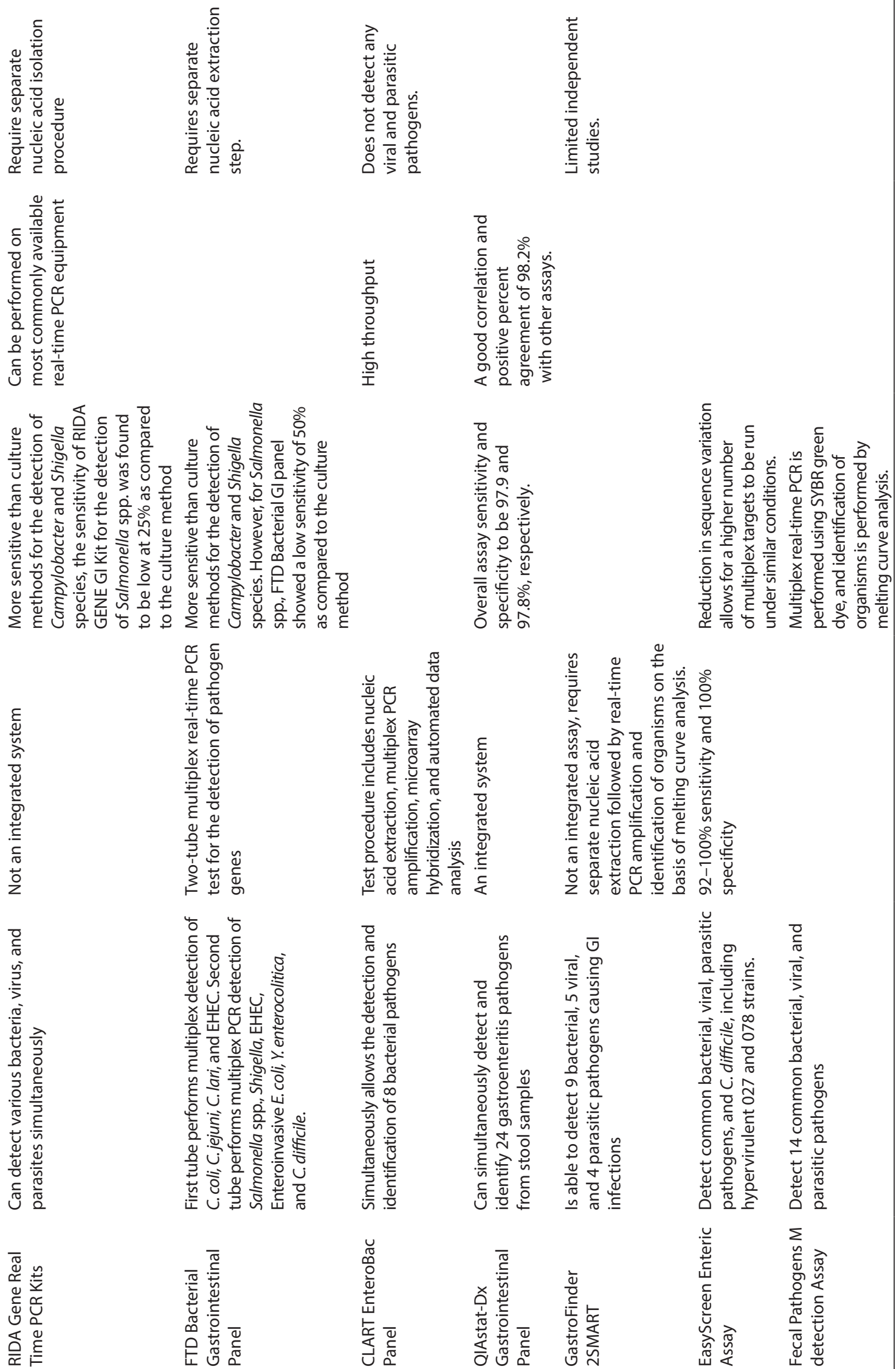

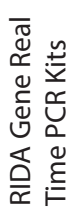
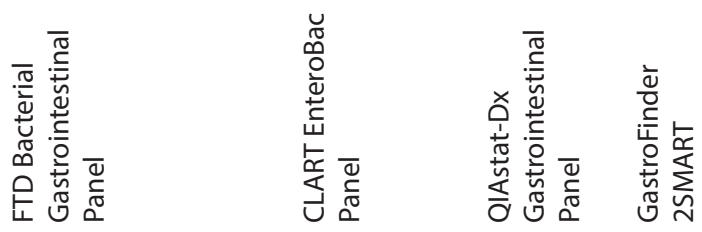


\section{Usage of Newer Techniques}

Gastroenteritis is not always severe, often resolving spontaneously and rapidly, thus raising the question of which patients presenting with Gl symptoms should undergo these tests. Considering the patient factors and cost-effectiveness is important in making this decision. Patient factors such as severity of symptoms and immune status have to be kept in mind. Also, rapid diagnosis is important in public health contexts as several enteropathogens can cause outbreaks and epidemics. With the shift of population dynamics toward older population, an advancement in treatment for patients with various life-threatening conditions of the past, such as hematological, rheumatologic, and oncological conditions, has led to an increase in people with immunocompromised status whose conditions can quickly deteriorate. These tests present us with the opportunity to identify the causative organism rapidly and adequately. Rapid molecular tests can also help us to differentiate the diarrhea due to host vs graft disease and that due to infectious etiology.

According to IDSA, ${ }^{52}$ the best approach to use these tests includes the following: The specimen with positive result on a culture-independent testing method should be made available for future testing purposes and culture to the laboratory.

- Future testing can then be done for the identification of species and determination of the serotype and further subtype by the molecular methods, like pulsed-field gel electrophoresis or whole-genome sequencing.

- Determination of the subtype further allows the detection of increased infections occurring due to a specific strain and hence facilitates in outbreak investigations by helping in finding a common exposure source for suspected case patients.

- Antimicrobial susceptibility can be done, which not only provides information about the drugs effective against that particular organism but also helps in the outbreak settings and in ongoing surveillance, which provides the local trends in resistance patterns as well as their mechanisms.

To conclude, we can surely say that these panel-based GI testing techniques are here to stay. Even though not all patients are likely to need these tests, in certain patients they could make the difference of life and death. Their use and interpretation of the result now depends on the use by the clinicians and their wisdom in making these panels a useful tool in diagnosis, without blindly treating for everything that comes positive.

\section{Future Aspects}

Judicious use of these culture-independent methods is beneficial for the patients as well as for clinicians. However, there are some lacunae yet to be filled by proper studies and improvement in technologies. One such issue is the lack of significant number of studies on the cost-effectiveness for the use of these assays that are needed to guide us. Another important issue is regarding the importance of mixed infections and their implication in patient presentation as well as treatment required. More studies are also required to study the role of quantitative diagnostics in regard to these assays. These assays can be used to study the epidemiology of diarrheal disease during vaccine efficacy trials to determine the relation to particular benefit of the vaccine. Another area where these assays might help us to understand is regarding mucosal immunology, of which our knowledge is limited.

\section{References}

1. da Cruz Gouveia MA, Lins MTC, da Silva GAP. Acute diarrhea with blood: diagnosis and drug treatment. J Pediatr (Rio J) 2020;96(1): 20. DOI: 10.1016/j.jped.2019.08.006.

2. Nemeth V, Pfleghaar N. Diarrhea. In: StatPearls [Internet]. Treasure Island (FL): StatPearls Publishing; 2020.

3. Tille PM. Bailey and Scott's diagnostic microbiology. 13th ed. Elsevier Publishers; 2013. p. 947.

4. Schiller LR. Chronic diarrhea evaluation in the elderly: IBS or something else? Curr Gastroenterol Rep 2019;21(9):45. DOI: 10.1007/ s11894-019-0714-5.

5. DuPont HL. Persistent diarrhea: a clinical review. JAMA 2016;315(24):2712-2723. DOI: 10.1001/jama.2016.7833.

6. Lucado J, Mohamoud S, Zhao L, et al. Infectious enteritis and foodborne illness in the United States, 2010 statistical brief \#150. Healthcare Cost and Utilization Project (HCUP) Statistical Briefs [Internet]. Rockville (MD): Agency for Health Care Policy and Research (US); 2013.

7. Tatte VS, Gopalkrishna V. Detection of different enteric viruses in children with diarrheal disease: evidence of the high frequency of mixed infections. Access Microbiol 2019;1(2):e000010. DOI: 10.1099/ acmi.0.000010.

8. Sattar SBA, Singh S. Bacterial gastroenteritis. In: StatPearls [Internet]. Treasure Island (FL): StatPearls Publishing; 2020.

9. Garcia LS, Arrowood M, Kokoskin E, et al. Practical guidance for clinical microbiology laboratories: laboratory diagnosis of parasites from the gastrointestinal tract. Clin Microbiol Rev 2018;31(1):e00025-e00017. DOI: 10.1128/CMR.00025-17.

10. Amjad M. An overview of the molecular methods in the diagnosis of gastrointestinal infectious diseases. Int J Microbiol 2020;81: 1-13. DOI: 10.1155/2020/8135724.

11. Giuliano C, Patel CR, Kale-Pradhan PB. A guide to bacterial culture identification and results interpretation. P 2019;44(4):192-200. PMID: 30930604; PMCID: PMC6428495.

12. Balsalobre-Arenas L, Alarcón-Cavero T. Rapid diagnosis of gastrointestinal tract infections due to parasites, viruses, and bacteria. Enferm Infecc Microbiol Clin 2017;35(6):367-376. DOI: 10.1016/j.eimc.2017.01.002.

13. Lee HM, Lee S, Lee BI, et al. Clinical significance of fecal lactoferrin and multiplex polymerase chain reaction in patients with acute diarrhea. Gut Liver 2015;9(5):636-640. DOI: 10.5009/gnl14106.

14. Barreda-García S, Miranda-Castro R, de-Los-Santos-Álvarez N, et al. Helicase-dependent isothermal amplification: a novel tool in the development of molecular-based analytical systems for rapid pathogen detection. Anal Bioanal Chem 2018;410(3):679-693. DOI: 10.1007/s00216-017-0620-3.

15. Buchan BW, Olson WJ, Pezewski M, et al. Clinical evaluation of a real-time PCR assay for identification of Salmonella, Shigella, Campylobacter (Campylobacter jejuni and C. coli), and Shiga toxinproducing Escherichia coli isolates in stool specimens. J Clin Microbiol 2013;51(12):4001-4007. DOI: 10.1128/JCM.02056-13.

16. Wohlwend N, Tiermann S, Risch L, et al. Evaluation of a multiplex real-time $P C R$ assay for detecting major bacterial enteric pathogens in fecal specimens: intestinal inflammation and bacterial load are correlated in campylobacter infections. J Clin Microbiol 2016;54(9):2262-2266. DOI: 10.1128/JCM.00558-16.

17. SobczykJ, Jain S, Sun X, et al. Comparison of multiplex gastrointestinal pathogen panel and conventional stool testing for evaluation of patients with HIV infection. Open Forum Infect Dis 2020;7(1):ofz547. DOI: 10.1093/ofid/ofz547.

18. Paitan $Y$, Roll TM, Adler A. Comparative performance study of six commercial molecular assays for rapid detection of toxigenic Clostridium difficile. Clin Microbiol Infect 2017;23(8):567-572. DOI: 10.1016/j.cmi.2017.02.016.

19. Monot $\mathrm{M}$, Eckert $\mathrm{C}$, Lemire $\mathrm{A}$, et al. Clostridium difficile: new insights into the evolution of the pathogenicity locus. Sci Rep 2015;5:15023. DOI: $10.1038 /$ srep15023. 
20. Yakob L, Riley TV, Paterson DL, et al. Mechanisms of hypervirulent Clostridium difficile ribotype 027 displacement of endemic strains: an epidemiological model. Sci Rep 2015;5:12666. DOI: 10.1038/srep12666.

21. McDonald LC, Gerding DN, Johnson S, et al. Clinical practice guidelines for Clostridium difficile infection in adults and children: 2017 update by the Infectious Diseases Society of America (IDSA) and Society for Healthcare Epidemiology of America (SHEA). Clin Infect Dis 2018;66(7):e1-e48. DOI: 10.1093/cid/cix1085.

22. Jensen MBF, Olsen KEP, Nielsen XC, et al. Diagnosis of Clostridium difficile: real-time PCR detection of toxin genes in faecal samples is more sensitive compared to toxigenic culture. Eur J Clin Microbiol Infect Dis 2015;34(4):727-736. DOI: 10.1007/s10096-014-2284-7.

23. Robilotti E, Deresinski S, Pinsky BA. Norovirus. Clin Microbiol Rev 2015;28(1):134-164. DOI: 10.1128/CMR.00075-14.

24. Yan $\mathrm{Y}$, Wang $\mathrm{H}-\mathrm{H}$, Gao L, et al. A one-step multiplex real-time RT-PCR assay for rapid and simultaneous detection of human norovirus genogroup I, II and IV. J Virol Methods 2013;189(2):277-282. DOI: 10.1016/j.jviromet.2013.02.004.

25. Ramanan P, Bryson AL, Binnicker MJ, et al. Syndromic panel-based testing in clinical microbiology. Clin Microbiol Rev 2018;31(1): 1-28. DOI: 10.1128/CMR.00024-17.

26. Huang RSP, Johnson $\mathrm{CL}$, Pritchard $\mathrm{L}$, et al. Performance of the Verigene ${ }^{\oplus}$ enteric pathogens test, Biofire FilmArray ${ }^{\mathrm{Tm}}$ gastrointestinal panel and LuminexxTAG ${ }^{\circledR}$ gastrointestinal pathogen panel for detection of common enteric pathogens. Diagn Microbiol Infect Dis 2016;86(4):336-339. DOI: 10.1016/j.diagmicrobio.2016.09.013.

27. Buss SN, Leber A, Chapin K, et al. Multicenter evaluation of the BioFire FilmArray gastrointestinal panel for etiologic diagnosis of infectious gastroenteritis. J Clin Microbiol 2015;53(3):915-925. DOI: 10.1128/ JCM.02674-14.

28. Wessels E, Rusman LG, van Bussel MJAWM, et al. Added value of multiplex luminex gastrointestinal pathogen panel (xTAG GPP) testing in the diagnosis of infectious gastroenteritis. Clin Microbiol Infect 2014;20(3):O182-O187. DOI: 10.1111/1469-0691.12364.

29. Claas EC. Performance of the XTAG gastrointestinal pathogen panel, a multiplex molecular assay for simultaneous detection of bacterial, viral, and parasitic causes of infectious gastroenteritis. J Microbiol Biotechnol 2013;23(7):1041-1045. DOI: 10.4014/jmb.1212.12042.

30. Navidad JF, Griswold DJ, Gradus MS, et al. Evaluation of LuminexxTAG gastrointestinal pathogen analyte-specific reagents for highthroughput, simultaneous detection of bacteria, viruses, and parasites of clinical and public health importance. J Clin Microbiol 2013;51(9):3018-3024. DOI: 10.1128/JCM.00896-13.

31. Yoo J, Park J, Lee HK, et al. Comparative evaluation of Seegene Allplex gastrointestinal, LuminexxTAG gastrointestinal pathogen panel, and BD MAX enteric assays for detection of gastrointestinal pathogens in clinical stool specimens. Arch Pathol Lab Med 2019;143(8):999-1005. DOI: 10.5858/arpa.2018-0002-OA.

32. KnabI L, Grutsch I, Orth-Höller D. Comparison of the BD MAX enteric bacterial panel assay with conventional diagnostic procedures in diarrheal stool samples. Eur J Clin Microbiol Infect Dis 2016;35(1): 131-136. DOI: 10.1007/s10096-015-2517-4.

33. DeBurger B, Hanna S, Powell EA, et al. Utilizing BD MAX enteric bacterial panel to detect stool pathogens from rectal swabs. BMC Clin Pathol 2017;17:7. DOI: 10.1186/s12907-017-0043-2.

34. Molling P, Nilsson P, Ennefors T, et al. Evaluation of the BD max enteric parasite panel for clinical diagnostics. J Clin Microbiol 2016;54(2): 443-444. DOI: 10.1128/JCM.02100-15.

35. Stokes W, Simner PJ, Mortensen J, et al. Multicenter clinical validation of the molecular BD max enteric viral panel for detection of enteric pathogens. J Clin Microbiol 2019;57(9):e00306-e00319. DOI: 10.1128/JCM.00306-19.
36. Amrud K, Slinger R, Sant N, et al. A comparison of the Allplex ${ }^{\mathrm{TM}}$ bacterial and viral assays to conventional methods for detection of gastroenteritis agents. BMC Res Notes 2018;11(1):514. DOI: 10.1186/ s13104-018-3645-6.

37. Coupland LJ, Mcelarney I, Meader E, et al. Simultaneous detection of viral and bacterial enteric pathogens using the Seeplex ${ }^{\oplus}$ Diarrhea ACE detection system. Epidemiol Infect 2013;141(10):2111-2121. DOI: $10.1017 /$ S0950268812002622.

38. Higgins RR, Beniprashad M, Cardona M, et al. Evaluation and verification of the Seeplex Diarrhea-V ACE assay for simultaneous detection of adenovirus, rotavirus, and norovirus genogroups I and II in clinical stool specimens. J Clin Microbiol 2011;49(9):3154-3162. DOI: 10.1128/JCM.00599-11.

39. Onori M,Coltella L,Mancinelli L, etal.Evaluation of a multiplexPCRassay for simultaneous detection of bacterial and viral enteropathogens in stool samples of paediatric patients. Diagn Microbiol Infect Dis 2014;79(2):149-154. DOI: 10.1016/j.diagmicrobio.2014.02.004.

40. Zboromyrska Y, Vila J. Advanced PCR-based molecular diagnosis of gastrointestinal infections: challenges and opportunities. Expert Rev Mol Diagn 2016;16(6):631-640. DOI: 10.1586/14737159.2016.1167599.

41. Valledor S, Valledor I, Gil-Rodríguez MC, et al. Comparison of several Real-Time PCR Kits versus a culture-dependent algorithm to identify enteropathogens in stool samples. Sci Rep 2020;10:4301. DOI: 10.1038/ s41598-020-61202-z.

42. Biswas JS, Al-Ali A, Rajput $P$, et al. A parallel diagnostic accuracy study of three molecular panels for the detection of bacterial gastroenteritis. Eur J Clin Microbiol Infect Dis 2014;33(11):2075-2081. DOI: 10.1007/s10096-014-2177-9.

43. Hannet I, Engsbro AL, Pareja J, et al. Multicenter evaluation of the new QIAstat Gastrointestinal Panel for the rapid syndromic testing of acute gastroenteritis. Eur J Clin Microbiol Infect Dis 2019;38(11): 2103-2112. DOI: 10.1007/s10096-019-03646-4.

44. Stark D, Garcia LS, Barratt JLN, et al. Description of Dientamoeba fragilis cyst and precystic forms from human samples. J Clin Microbiol 2014;52(7):2680-2683. DOI: 10.1128/JCM.00813-14.

45. Besser JM, Carleton HA, Trees E, et al. Interpretation of wholegenome sequencing for enteric disease surveillance and outbreak investigation. Foodborne Pathog Dis 2019;16(7):504-512. DOI: 10.1089/fpd.2019.2650.

46. Franco-Duarte $R$, Černáková $L$, Kadam $S$, et al. Advances in chemical and biological methods to identify microorganismsfrom past to present. Microorganisms 2019;7(5):130. DOI: 10.3390/ microorganisms7050130.

47. Chin CS, Sorenson J, Harris JB, et al. The origin of the Haitian cholera outbreak strain. N Engl J Med 2011;364(1):33-42. DOI: 10.1056/ NEJMoa1012928.

48. Joensen KG, Engsbro AL, Lukjancenko O, et al. Evaluating nextgeneration sequencing for direct clinical diagnostics in diarrhoeal disease. Eur J Clin Microbiol Infect Dis 2017;36(7):1325-1338. DOI: 10.1007/s10096-017-2947-2.

49. Wang S, Hong W, Dong S, et al. Genome engineering of Clostridium difficile using the CRISPR-Cas9 system. Clin Microbio Infect 2018;24(10):1095-1099. DOI: 10.1016/j.cmi.2018.03.026.

50. Vinayak S, Pawlowic MC, Sateriale A, et al. Genetic modification of the diarrhoeal pathogen Cryptosporidium parvum. Nature 2015;523(7561):447-480. DOI: 10.1038/nature14651.

51. Beverley SM. CRISPR for Cryptosporidium. Nature 2015;523(7561): 413-414. DOI: 10.1038/nature14636.

52. Imdad A, Retzer F, Thomas LS, et al. Impact of culture independent diagnostic testing on recovery of enteric bacterial infections. Clin Infect Dis 2018;66(12):1892-1898. DOI: 10.1093/cid/cix1128. 